\title{
Validación del modelo de modernización para la gestión de las organizaciones (MMGO) Versión 10*
}

\author{
Validation of the Modernization Model for \\ the Management of Organizations (MMMO) \\ Version 10
}

\section{Validação do modelo de modernização para a gestão das organizações (MMGO) Versão 10}

\section{Carlos Andrés Plaza Cárdenas ${ }^{* *}$}

\section{Fecha de recibido: 11 de febrero de 2015}

Fecha de aprobado: 29 de julio de 2015

Doi: dx.doi.org/10.12804/rev.univ.empresa.30.2016.03

Para citar este artículo: Plaza Cárdenas, C. A. (2016). Validación del modelo de modernización para la gestión de las organizaciones (MMGO) versión 10. Universidad \& Empresa, 18(30), 55-73. Doi: dx.doi. org/10.12804/rev.univ.empresa.30.2016.03

\section{RESUMEN}

El modelo de modernización para la gestión de las organizaciones (MMGO) se hizo público, de manera amplia, en el año 2011. Es necesario refrescar sus componentes y actualizarlo, para que se adecúe al siempre cambiante entorno de las pyme en Colombia, sin perder su esencia y sin desconocer los resultados de investigaciones anteriores relativas al mismo. De esto da cuenta la investigación que aquí se presenta. El grupo G3Pymes de la Universidad

* Este artículo es producto de un proyecto de investigación aprobado y financiado por la universidad EAN (Bogotá). Esta institución asignó al proyecto un presupuesto de \$3.785.600, además de un importante apoyo en cabeza de la Vicerrectoría de Investigaciones y de algunos profesores que contribuyeron con su asesoría para el adecuado desarrollo del mismo.

** Estudiante de la Maestría en Gestión de Organizaciones, de la Universidad EAN (Bogotá) y la Université du Québec à Chicoutimi (UQAC) (Canadá). Investigador vinculado al grupo G3Pymes de la Universidad EAN (Bogotá). Correo electrónico: cplazaca5931@ean.edu.co 
EAN, en el que se inscribe este proyecto, innova constantemente para desarrollar el modelo, que se encuentra en la versión 10, con la actualización de tres de sus componentes: análisis del entorno, comunicación e información y responsabilidad social.

Palabras clave: actualización, componentes, MMGO, validación, variables.

\section{ABSTRACT}

The Modernization Model for the Management of Organizations (MMMO) was widely made public in 2011. It is now necessary to refresh its components and update it so that it will adapt to the ever changing environment of SME in Colombia without its losing its essential nature or ignoring the results of previous investigations about it. It is taken into account in the research presented herein. This work has been done in order to fulfill this need. The G3Pymes Group of EAN University, with which this project is registered, constantly innovates in the development of the model. At present, the model is in version 10 in which three of its components are updated: environmental analysis, communication and information and social responsibility.

Keywords: Updating, components, MMGO, validation, variables.

\section{RESUMO}

O modelo de modernização para a gestão das organizações (MMGO) foi feito público, de maneira ampla, no ano 2011. É preciso agora refrescar as suas componentes e atualizá-lo para que este se adeque ao sempre mutável entorno das MPEs na Colômbia. Isto sem perder a sua essência e sem desconhecer os resultados de pesquisas anteriores relativas ao mesmo. Disto da conta a pesquisa que aqui se apresenta. Este trabalho se tem realizado com o fim de satisfazer esta necessidade. O grupo G3Pymes da Universidade EAN, no que se inscreve este projeto, inova constantemente para desenvolver o modelo. Atualmente, este se encontra na versão 10, na que se atualizam três das suas componentes: análise do entorno, comunicação e informação e responsabilidade social.

Palavras-chave: atualização, componentes, MMGO, validação, variáveis. 


\section{INTRODUCCIÓN}

Consciente de que el desarrollo económico en el mundo globalizado depende en gran medida de la creación y la modernización de las pequeñas y medianas empresas (pymes) productivas y de servicios, la Universidad EAN (Bogotá) ha hecho importantes esfuerzos para apoyar los procesos de modernización de este tipo de organizaciones en este país (Escuela de Administración de Negocios [EAN], 2014).

Entre otras acciones, el grupo de investigación G3Pymes de la Universidad EAN, desarrolló el modelo para la modernización de la gestión de las organizaciones (MMGO). Este se encuentra en la versión 9D e incorpora dieciséis componentes considerados en cuatro estadios. Permite definir la situación de una pyme en el momento de su aplicación, al clasificar cada componente en un estadio y, además, facilita visualizar las acciones necesarias para mejorar y pasar al siguiente nivel (Pérez Uribe et al., 2009).

El actual mundo globalizado y el entorno siempre cambiante que este plantea para las organizaciones hacen que el MMGO deba renovarse, con el fin de propiciar la obtención de resultados eficientes en su aplicación. En atención a esta necesidad, el proyecto de investigación se ocupa de la validación de la versión 10 del modelo, en la que se actualizan tres de sus componentes: análisis de entorno, comunicación e información y responsabilidad social empresarial.

En efecto, la nueva versión del MMGO se ocupa de estos tres elementos como una fase intermedia para hacer una actualización definitiva e integral del MMGO. Con el propósito de validar estos tres nuevos factores, es necesario aplicar la nueva versión a una pyme. Se seleccionó como terreno de investigación una ubicada en la ciudad de Bogotá. Luego de su aplicación, es necesario diligenciar el modelo en su versión 9D, para analizar el impacto de los mencionados tres componentes en el informe integral.

Tras implementar los modelos en sus versiones v10 y 9D se apreció que los datos recolectados para análisis de entorno (v10) no son suficientemente significativos, si se equiparan con el mismo componente en la versión anterior (9D). Los elementos comunicación e información y responsabilidad social empresarial de la nueva versión resultan ser mejores, en particular, en la significancia de la información que proporcionan y en la agilidad de su aplicación. Estos detalles y los aspectos comparativos que dan lugar 
a estos planteamientos se presentarán de forma detallada en este reporte final de la investigación, el cual está estructurado en cinco secciones: 1) planteamiento del problema; 2) contexto y marco teórico; 3) metodología; 4) resultados y discusión, y 5) conclusiones.

\section{PLANTEAMIENTO DEL PROBLEMA}

La realidad de las organizaciones es siempre cambiante. Para que pueda ser sensible a la misma, una propuesta teórico-práctica como el modelo para la modernización de la gestión de las organizaciones requiere estar actualizándose. Esto para que evolucione a la par del entorno $\mathrm{y}$, de este modo, sirva a las necesidades de las empresas para las cuales fue concebido, es decir, las pyme.

\section{CONTEXTO Y MARCO TEÓRICO}

\subsection{El entorno de las tecnologías de la información y la comunicación (TIC) en Colombia}

Existe una clara tendencia a fomentar la competencia en mercados de telecomunicaciones y, en general, en todos aquellos vinculados con las TIC. Según la Unión Internacional de Telecomunicaciones ([UIT], 2011), el nuevo grado de eficiencia del mercado se debe a que varios países se han encargado de atraer operadores que ofrecen distintos portafolios de servicio a los usuarios. La intensificación de la competencia para 2010 en todo el mundo alcanzó un porcentaje de $94 \%$ para la tecnología $3 \mathrm{G}$ y, para la de $2 \mathrm{G}$, pasó de $79 \%$ en 2010 a $90 \%$ en 2011 (UIT, 2011).

Se estima que las cifras mencionadas seguirán creciendo, debido, por una parte, a la presencia de tecnologías como 4G-LTE y, por otra, a las crecientes necesidades de los usuarios de contar con un ancho de banda cada vez mayor para hacer uso tanto de redes sociales como de toda la gama de aplicaciones y servicios que a diario se ponen a disposición. La influencia de esta tendencia es clara, ya que nuevos actores en el mercado y una mayor competencia significan, para empresas del sector comola seleccionada(ver sección 3), la necesidad de ampliar su actual base de clientes y de contar con más preparación para enfrentar estándares de competitividad superiores (UIT, 2011).

Para cumplir con su rol en esta materia, el Gobierno colombiano regula a los proveedores de telecomunicaciones, con el fin de lograr que sus objetivos sean la calidad y la masificación, para llegar a zonas apartadas 
sin que esto signifique incurrir en costos muy elevados. En el momento de redactar este artículo, cursa su trámite un proyecto en el Congreso de la República que busca unificar las políticas del país en cuanto al despliegue de redes y sistemas de información (Dinero, 2014).

Por otro lado, tanto la industria vinculada con las TIC como el Ministerio de Tecnologías de la Información y las Comunicaciones (MinTIC) están enfocados en promover la digitalización en las pyme, por medio de programas como Mypime digital. Sin embargo, según el presidente de la Cámara Colombiana de Informática y Telecomunicaciones (CCIT) "un tercio de las pyme colombianas no usan tecnología y mucho menos se conecta a Internet a través de dispositivos móviles" (Dinero, 2014). Aunque para el Gobierno esto da cuenta de un escenario negativo, el mismo fenómeno puede observarse con optimismo, como la existencia de un importante potencial de crecimiento e innovación para las pyme en esta materia.

En Colombia, se ejecutan acciones concretas como 1) la expedición de la Ley 1341 del 30 de julio de 2009, "Por la cual se definen principios y conceptos sobre la sociedad de la información y la organización de las Tecnologías de la Información y las
Comunicaciones - TIC - , se crea la Agencia Nacional del Espectro y se dictan otras disposiciones"; 2) la creación de la Comisión de Regulación de Telecomunicaciones; 3) la instauración del Fondo de Tecnologías de la Información y las Comunicaciones, y 4) el lanzamiento de la estrategia del Gobierno denominada FITI: fortalecimiento de la industria de tecnologías de la información, que trata de dinamizar líneas de acción que conforman un modelo integral (MinTIC, 2013a). Estas forman parte de una serie de iniciativas de promoción del sector de las TIC que se están llevando a cabo desde ocho dimensiones: 1) visión y estrategia del sector; 2) investigación, desarrollo e innovación (I+D+i);3) emprendimiento y fortalecimiento empresarial; 4) talento humano; 5) calidad; 6) normatividad; 7) asociatividad, y 8) infraestructura. Estas iniciativas son una muestra del ambiente que está generando el Gobierno, con el propósito de atraer la inversión al país en lo concerniente a este sector.

Para el sector de las TIC es muy importante la mano de obra calificada. El recurso humano es indispensable para asegurar su crecimiento. Sin embargo, en temas de innovación, no se cuenta con la estructura y el capital humano necesarios (Portafolio, 2013). En consonancia, la situación 
laboral de los empleados del sector es estable y se identifica un muy bajo índice de desempleo en el mismo (2\%) (MinTIC \& Federación Nacional de Software y Tecnologías de la Información [Fedesoft], 2012). Esto muestra su capacidad para atraer al talento humano disponible en el mercado laboral. La única falencia identificada es la alta rotación de los profesionales. La mayoría de profesionales $(50,31 \%)$ no lleva más de dos años en el cargo que hoy ocupa y solo una minoría (menos de $10 \%$ ) está en el mismo desde hace más de diez años (MinTIC \& Fedesoft, 2012).

Según la CCIT, existe un déficit amplio de profesionales en tecnología que aumenta cada año. El sector de las TIC se da el lujo de crecer a un ritmo superior al de los demás campos de la economía, pues cada año aumenta su participación en el PIB (Portafolio, 2013). En el mercado laboral, sus profesionales están entre aquellos que más rápido obtienen trabajo, con un tiempo promedio menor a un año. Se estima que se requieren alrededor de 12.000 graduandos cada año y, por ahora, solo se cuenta con 5.000. El Gobierno está tratando de disminuir este déficit con planes como las convocatorias de talento digital, las cuales son impulsadas periódicamente por el MinTIC (2013b).
En 2011, Colombia registró una brecha, materializada en una carencia de profesionales, que alcanzó un $25 \%$. Se estima que esta aumente hasta un $33 \%$ en 2015 (Martín, 2013). Esto significa que este país podría pasar de una falta de 6.391 profesionales en tecnologías de la información a uno de casi el doble (12.403) en 2015 (Martín, 2013).

El tema laboral es un asunto de vital importancia para el sector en este país y en los demás que forman parte de la región en donde se encuentra. De acuerdo con Jordi Botifoll, vicepresidente senior de Cisco para América Latina:

Una mano de obra capacitada es una ventaja competitiva para los países de la región, para la economía basada en conocimientos del siglo XXI. En la medida en que América Latina experimenta la emergencia rápida de tendencias tecnológicas como la $\mathrm{Nu}$ be, movilidad, video e Internet de todo, esta brecha de profesionales capacitados presenta un desafío real para el desarrollo económico de la región. Sin los conocimientos adecuados, el progreso tecnológico no se traducirá en aumentos en productividad (Martín, 2013, p. 1).

Otra limitante del sector es que no existe un claro sistema de contratación. Además, no se diferencia adecuadamente entre el profesional y el tecnólogo y, de hecho, múltiples 
empresas no están contratando los perfiles que necesitan. Según una encuesta reciente de Fedesoft, los tecnólogos solo representan el 4,2\% del total de trabajadores de la industria de software, mientras los técnicos profesionales representan apenas un $0,95 \%$ y los técnicos laborales, un 0,48\% (Hernández, 2014).

\subsection{MMGO versión 10}

El MMGO es una herramienta utilizada desde el año 2003 por parte del grupo de investigación G3Pymes de la Universidad EAN (Pérez, Garzón $\&$ Nieto, 2009). Surge de la consideración de las múltiples necesidades de las pyme y, en particular, del sinnúmero de dificultades que enfrentan cuando tratan de aplicar modelos de gestión diseñados para grandes empresas, en lo fundamental, de países desarrollados (Pérez et al., 2009).

Este modelo ha sido aplicado en más de 127 pyme en Colombia. Esta herramienta, como ya se ha indicado, tiene dieciséis componentes y señala cuatro estadios. El MMGO, en su actual versión 10, define la situación en la que se encuentra la pyme en el momento de su aplicación, al clasificar cada componente en uno de los cuatro estadios (del 1 al 4). Esto permite visualizar las acciones necesarias para mejorar en el con- texto organizacional (Pérez Uribe et al., 2009). Los componentes de este modelo se presentan a continuación (G3Pymes, 2013):

1. Análisis del entorno: para hacer inteligible la relación de la organización con su entorno, desde el punto de vista de la gestión organizacional, es posible considerar cinco variables básicas, que cubren aspectos susceptibles de afectar directa o colateralmente a la organización desde la función gerencial. Estas son: 1) reconocimiento del terreno competitivo; 2) inteligencia competitiva; 3) órganos de regulación de los efectos externos; 4) mecanismos de respuesta e influencia, y 5) apalancamiento para gestionar el entorno.

2. Direccionamiento estratégico: un modelo de direccionamiento estratégico agrega valor a las operaciones que requiere estar acompañado por una estructura organizativa adecuada y un sistema de valores de todos los miembros de la organización. Este último, a su vez, debe permitir el alineamiento de la estrategia con el sistema de finalidades. Para una compañía, es clave tener un esquema formal de planeación orientado a la gestión, al desempeño y al control, acompañado de un sistema de indicadores que 
permita monitorear los objetivos planteados y facilite el aprendizaje organizacional y la gestión del conocimiento.

3. Gestión de mercadeo: la matriz de mercadeo explora las actividades de gestión de mercados y comercialización realizadas por la empresa. En la calificación que se obtiene son importantes aspectos como la implantación de procesos formales de estudio, el análisis de los mercados y segmentos, el reconocimiento de un producto con sus atributos de mercado, la promoción de los productos en los segmentos objetivo, el análisis formal de los precios, la organización de sistema de distribución y la realimentación que se obtenga de clientes y distribuidores.

4. Cultura organizacional: examina el alcance y la profundidad con los que se desarrolla, involucra y apoya a las personas para que participen en el mejoramiento de la calidad de vida en la compañía. Determina si se fomenta en los individuos la autonomía, la creatividad y la capacidad de colaborar y comprometerse activamente con el mejoramiento de la empresa, así como con sus procesos, productos y servicios. También se revisan los esfuerzos de la pyme por construir y mantener un ambiente que propicie la participación real, el trabajo en equipo y el respeto por las personas.

5. Estructura organizacional: evalúa el proceso que adelanta la empresa para establecer las relaciones formales entre el personal, las competencias y los factores físicos. Considera también la relación y alineamiento de la estructura organizacional con la estrategia de la compañía y los sistemas de información utilizados para facilitar la toma de decisiones.

6. Producción: se valora la gestión de producción en los aspectos de planeación y proyección de los recursos productivos como una actividad esencial para satisfacer los requerimientos del cliente.

7. Gestión financiera: la matriz planteada muestra los estadios en los cuales se ubica la empresa y califica las variables de inversión, financiación y rentabilidad como fundamentales. Sustenta la observación en los elementos y medios desarrollados o utilizados por la compañía, los procesos definidos y la información producida, su calidad y divulgación.

8. Gestión humana: este componente organizacional examina el desarrollo y seguimiento de 1) un plan estratégico de gestión humana; 2) las actividades en esta materia, necesarias para el eficaz 
ingreso de los trabajadores a la empresa; 3) los esfuerzos para promover el mejoramiento de los conocimientos y las destrezas para un trabajo determinado y para medir técnicamente el desempeño de cada trabajador; 4) las actividades para el manejo salarial y la satisfacción de las necesidades primarias de los trabajadores y sus familias; 5) las tareas para cumplir de manera estricta con el régimen laboral, las Leyes 100 y 50 ; 6) los programas, las políticas y las actividades para el cumplimiento de las disposiciones legales que están reguladas por el régimen laboral colombiano sobre salud ocupacional y seguridad industrial, y 7) una serie de acciones tendientes a desarrollar, involucrar y apoyar a las personas para que participen en el mejoramiento de la calidad de vida, colaboren y se comprometan con el mejoramiento de la calidad de la empresa, sus procesos, productos y servicios.

9. Exportaciones: se evalúan la existencia de una estrategia de exportaciones definida y explícita, los insumos de información sobre los mercados externos, la presencia de productos y servicios exportables y la forma en que la empresa mide y recibe realimentación de dichos mercados para la toma de decisiones interna.
10. Importaciones: se revisan la existencia de una estrategia de importaciones definida y explícita, los insumos de información sobre proveedores externos, la existencia de insumos importables y cómo la empresa mide y recibe realimentación de esos proveedores para la toma de decisiones interna.

11. Logística: en este aspecto se examina la capacidad logística de la organización. La planeación y proyección de los recursos productivos es una labor esencial para satisfacer los requerimientos del cliente. Por esto, la logística se convierte en un concepto complementario clave para competir. Las relaciones con los proveedores y los clientes son mejoradas gracias a la logística. La tecnología y el sistema de costos son garantes de una adecuada gestión del sistema de operaciones.

12. Asociatividad: el objetivo de esta matriz es estimar el potencial asociativo de las pyme en diversas modalidades de asociatividad. Estas organizaciones se asocian y conforman redes empresariales que constituyen la base productiva de una Nación. El modo como se relacionan no se puede establecer a priori, pero se ha encontrado que uno de los criterios básicos es la capacidad 
que tiene cada una para brindar un valor agregado a las demás en una estrategia asociativa.

13. Comunicación e información: este componente está orientado a medir dos grandes variables fundamentales para la gestión de la comunicación e información: 1) conversaciones en los ámbitos de la comunicación empresarial, y 2) gestión documental. Estas se vuelven tangibles en el uso del lenguaje, de los medios de comunicación y las actuaciones empresariales.

14. Innovación y conocimiento: la aplicación de la matriz al componente de conocimiento e innovación pretende calificar la situación de la empresa en el uso y la producción de conocimientos nuevos de todo tipo, los cuales pueden llegar a hacerla más competitiva. En la calificación son importantes 1) la existencia de un plan formal para incorporar conocimiento a la empresa en todas su áreas y para utilizarlo; 2) la presencia de personas con habilidades para manejar estos conocimientos, y 3) la forma como están organizadas y su participación en grupos de trabajo para la toma de decisiones. Interesa reconocer también los métodos y procesos para generar y utilizar conocimientos en la compañía y las formas de medir el desempeño de las personas y las áreas de la empresa respecto a la innovación. Por último, intenta identificar elementos de una cultura de innovación en la organización.

15. Responsabilidad social empresarial: permite evaluar las políticas y actividades relacionadas con el enfoque estratégico, la gestión y el impacto de la responsabilidad social empresarial. Asimismo, se consideran los registros y documentos que sirven de soporte al sistema para examinar su implementación.

16. Gestión ambiental: revisa las políticas de la selección y del uso de materias primas, la optimización de procesos, el análisis del ciclo de vida del producto y la presencia de residuos sólidos, aguas residuales, emisiones atmosféricas, olores, ruido, publicidad exterior visual, salud y seguridad industrial. Se toman en cuenta los registros y documentos que sirven de soporte al sistema para evaluar su implementación, las actividades para el mejoramiento ambiental y la organización del departamento de gestión ambiental (DGA).

\section{METODOLOGÍA}

Para la investigación vinculada con la validación de la versión 10 del 
MMGO se utilizó como terreno de investigación una pyme que se denomina Compusistems. Este es un pseudónimo manejado en el trabajo, con el fin de guardar el anonimato de la organización y la confidencialidad de la información suministrada.

Los datos empleados resultan de una aplicación efectiva del MMGO en esta organización. Se hizo un acercamiento a esta pyme bogotana que opera en el sector de las TIC para implementar el modelo. Se observaron todos los componentes del MMGO en sus dos versiones. El uso del método comparativo permitió hacer un análisis de la información y decantar los avances y retrocesos y, de este modo, hacer la validación de la versión 10 de esta herramienta.

\section{RESULTADOS Y DISCUSIÓN}

En procura de validar los tres componentes del MMGO ya mencionados (análisis del entorno, comunicación e información y responsabilidad social empresarial), se hizo una aplicación en la empresa Compusistems tanto de la versión más reciente (v10) de la herramienta como de la anterior (v9D), para obtener los resultados exactos de cada versión y comparar su impacto en el resultado global del modelo. Más adelante, se validó cada componente, se analizó su pertinencia con referencia en sus semejantes en la versión 9D y se emitió un concepto acerca de los aspectos que se pueden ajustar o aprobar, según el caso.

\subsection{Resultados del informe integral del MMGO en sus dos versiones}

Luego de aplicar los modelos en sus dos versiones, fue posible obtener resultados para la investigación, los cuales se muestran a continuación. Estos son un punto de partida necesario para la validación del MMGO v10 y posibilitan hacer comparativos mediante el análisis del informe integral. De esta forma, se hizo posible evaluar si, con la misma información y situación de la empresa Compusistems, los tres componentes actualizados alteran el estadio en el que se encuentra definida la organización. A continuación, en las figuras 1 y 2 , se exponen los informes integrales obtenidos.

En este informe integral, cuyos resultados básicos se incorporan en la figura 1, se aprecia que Compusistems está ubicado en el estadio 2 , debido a que esta organización obtiene un porcentaje de $33,6 \% \mathrm{y}$, según el MMGO, las empresas se encuentran en estadio 1 si su ponderación está entre $0 \%$ y $25 \%$; estadio 2 entre $25,1 \%$ y $50 \%$, estadio 3 entre $50,1 \%$ y $75 \%$ y estadio 4 si es superior a $75,1 \%$. 
Figura 1. Informe integral de la aplicación del MMGO v.9D

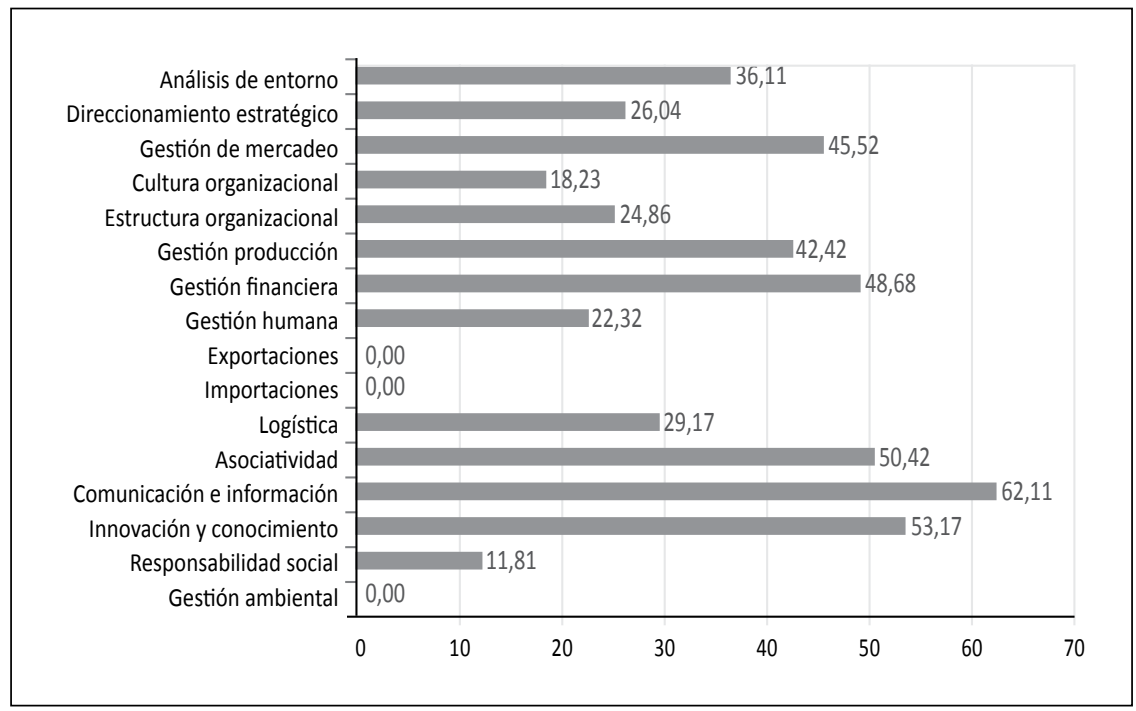

Fuente: elaboración propia.

Los principales componentes sus- dad social, la cultura organizacioceptibles demejora, es decir, aquellos nal, la gestión humana, la estructura que han resultado con una califica- organizacional y el direccionamiención muy baja, son la responsabili- to estratégico.

Figura 2. Informe integral de la aplicación del MMGO v10

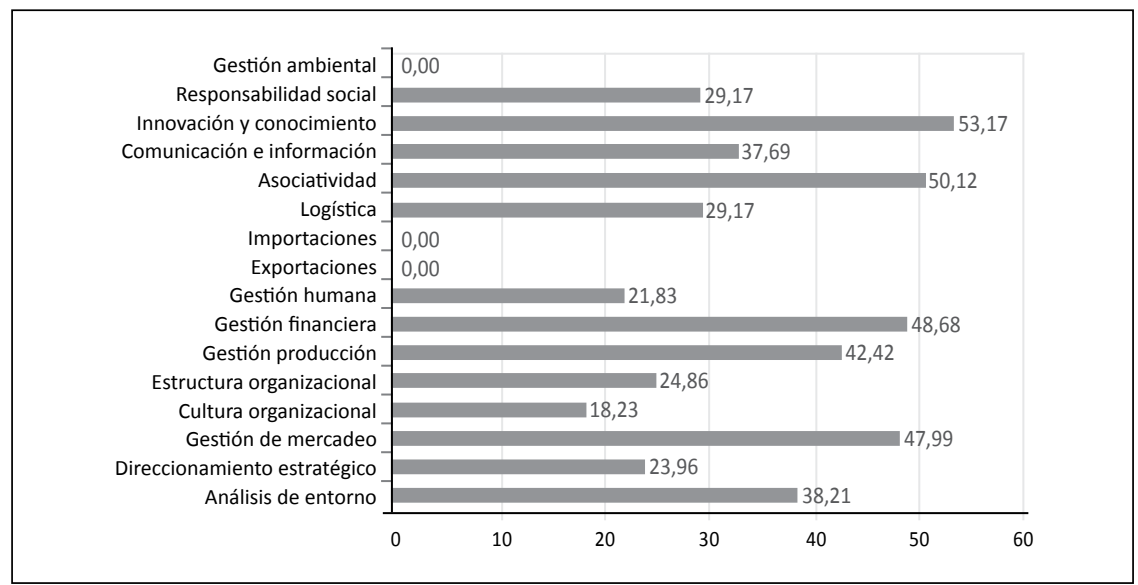

Fuente: elaboración propia. 
El informe integral contiene los tres componentes que han sido actualizados (figura 2). El estadio de la empresa continúa siendo el mismo (2) y, como se indicó, esto señala que la empresa tiene un enfoque hacia los procesos. Este aspecto no se ve alterado por la aplicación de esta nueva versión del MMGO.

La ponderación promedio de todos los componentes del informe integral del MMGO v10 es de 32,9\%, lo que marca una variación de solo 0,7 puntos. Este dato no es muy diciente y resalta la importancia de llevar a cabo un análisis individual de los tres componentes, que son objeto de estudio destacado en este trabajo. Esto se hará más adelante.

Por otra parte, se evidencian algunos cambios en el análisis de los informes integrales. El componente de comunicación e información no es uno de los fuertes en los resultados de la versión 10. Respecto a los componentes susceptibles de mejora, la responsabilidad social, que era el componente calificado en la versión 9D como el más débil, deja de convertirse en un aspecto crítico en la versión 10 y sale de la lista de los principales componentes débiles de la organización (tabla 1).

Tabla 1. Variación en los componentes objetos de estudio

\begin{tabular}{|l|c|c|}
\hline \multicolumn{1}{|c|}{ Componentes } & MMGO v9D & MMGO v10 \\
\hline Análisis de entorno & 36,11 & 38,21 \\
\hline Comunicación e información & 62,11 & 32,69 \\
\hline Responsabilidad social & 11,81 & 28,17 \\
\hline
\end{tabular}

Fuente: elaboración propia.

Se observa que la variación de los nuevos componentes no es muy representativa como para cambiar el estadio en el que se encuentra. Sin embargo, la variación de los resultados es muy significativa, al punto de modificar la relevancia de estos componentes y dejar de catalogar la comunicación e información como fortaleza o la responsabilidad social como debilidad. Se aprecia que el resultado numérico del componente análisis de entorno es similar en las dos aplicaciones (versión anterior y nueva).

\subsection{Resultados individuales de los tres componentes}

Para entender las causas de las variaciones señaladas en los resultados de la aplicación de las dos versiones es necesario profundizar en el análisis. Esto conduce a revisar las varia- 
bles de cada uno de estos componentes, no solo para validar sus resultados numéricos, sino también para evaluar la información que cada uno está considerando.

\subsubsection{Componente de análisis de entorno}

En la versión 10, este componente propone cinco variables principales que cubren aspectos susceptibles de afectar directa o colateralmente a la organización desde la función gerencial (tabla 2). Estos cinco factores son: 1) reconocimiento del terreno competitivo; 2) inteligencia competitiva; 3) órganos de regulación de los efectos externos; 4) mecanismos de respuesta e influencia, y 5) apalancamiento para gestionar el entorno (G3Pymes, 2013).

Tabla 2. Resumen de las variables del componente de análisis de entorno v10

\begin{tabular}{|l|c|}
\hline $\begin{array}{l}\text { Reconocimiento del terreno competitivo: mapa de jerarquías estratégicas (interacciones } \\
\text { con otras organizaciones). }\end{array}$ & 47,22 \\
\hline $\begin{array}{l}\text { Reconocimiento del terreno competitivo: mapas del entorno organizacional (lugar en la } \\
\text { industria). }\end{array}$ & 29,17 \\
\hline Reconocimiento del terreno competitivo: alineación estratégica (el norte de la organización). & 13,89 \\
\hline Inteligencia competitiva: planear la siguiente jugada (insumos). & 58,33 \\
\hline Inteligencia competitiva: planear la siguiente jugada (capacidades estratégicas). & 50,00 \\
\hline Inteligencia competitiva: planear la siguiente jugada (indicadores de gestión). & 33,33 \\
\hline Órganos de regulación de los efectos externos (impacto desde los clientes). & 37,50 \\
\hline Órganos de regulación de los efectos externos (impacto desde las instituciones del entorno). & 45,24 \\
\hline Mecanismos de respuesta e influencia (capacidad de respuesta). & 29,17 \\
\hline Mecanismos de respuesta e influencia (capacidad de influencia). & 30,56 \\
\hline Apalancamiento para gestionar el entorno: técnico (incluye tecnologías duras y blandas). & 54,17 \\
\hline Apalancamiento para gestionar el entorno: financiero. & 37,50 \\
\hline
\end{tabular}

Fuente: elaboración propia.

En la versión 9D, este componente analiza cuatro niveles de entorno: meta, macro, meso y micro (tabla 3 ). En el metaentorno se ubican las políticas de carácter más general, las cuales rebasan el período de un
Gobierno y se convierten en políticas de Estado y Nación. Estas conducen a la aceptación de objetivos comunes $\mathrm{y}$ al direccionamiento de esfuerzos conjuntos para alcanzar dichos objetivos. En cuanto al entorno macro- 
económico, existe consenso en que los cambios que se producen en este, en un mundo globalizado, afectan de forma cada vez más directa el desempeño de las organizaciones.

Desde la perspectiva del mesoentorno, la consolidación de asociaciones y alianzas empresariales, que permitan aprovechar las fortalezas de los participantes y abordar tareas inalcanzables para una organización individualmente considerada, es una condición necesaria para el desarrollo de las empresas.

Desde el punto de vista de la organización (nivel micro), la competitividad depende de su capacidad de entenderse como un todo -integral - y de empezar a gestionar su conocimiento para conseguir un adecuado posicionamiento y una mayor rentabilidad (G3Pymes, 2009).

Tabla 3. Resumen de variables del elemento de análisis de entorno v9D

\begin{tabular}{|l|c|}
\hline Entorno global meta & 33,33 \\
\hline Entorno país (macro), políticas macroeconómicas y resultados actuales y proyectados & 25,00 \\
\hline Entorno sectorial y regional (meso) & 16,67 \\
\hline Entorno cercano o próximo (grupos de interés —stakeholders—excepto clientes) & 33,33 \\
\hline Cadenas productivas y clúster & 41,67 \\
\hline Magnitud y comportamiento del mercado de la cadena & 41,67 \\
\hline Competencia/organizaciones similares & 33,33 \\
\hline Producto o servicio & 41,67 \\
\hline Precio o tarifa & 58,33 \\
\hline
\end{tabular}

Fuente: elaboración propia.

Aunque luego de la aplicación de las dos versiones del MMGO en la misma organización, en el mismo tiempo y con la misma información se obtuvieron resultados numéricos similares, la calidad de los datos obtenidos con la versión 9D fue mejor que aquella lograda con la implementación de la versión 10 , pues a pesar de que la cantidad de variables en el componente es similar, el enfoque cambia bastante.

El componente de la versión más reciente no contempla todos los aspectos del entorno y da la impresión de que excluye algunos detalles en el proceso de recolección de información. La dinámica que se maneja en la versión 9D desde el metaentorno 
hasta el microentorno resulta más sencilla y práctica tanto en su aplicación como en el análisis de la información, sin omitir detalles relevantes del entorno.

Por lo anterior, tras aplicar y comparar este factor en sus diferentes versiones, desde el punto de vista práctico y experimental, se considera que la actualización del componente no es viable y, en consecuencia, se recomienda continuar utilizando el de la versión 9D.

\subsubsection{Componente de comunicación e información}

En la versión 10, este componente está orientado a medir dos grandes variables fundamentales para la gestión de la comunicación e información: 1) conversaciones en los ámbitos de la comunicación empresarial, y 2) gestión documental (tabla 4). Estas se hacen visibles en el uso del lenguaje, de los medios de comunicación y en las actuaciones empresariales (G3Pymes, 2013).

Tabla 4. Resumen de variables del componente comunicación e información V10

\begin{tabular}{|l|c|}
\hline $\begin{array}{l}\text { Conversaciones en los ámbitos de la } \\
\text { comunicación empresarial }\end{array}$ & 45,14 \\
\hline $\begin{array}{l}\text { Gestión documental en ámbitos de } \\
\text { la comunicación empresarial }\end{array}$ & 20,24 \\
\hline
\end{tabular}

Fuente: elaboración propia.
En la versión 9D, el componente está orientado a medir tres variables: 1) reuniones; 2) registro de datos, y 3) creación y transmisión de conocimientos (tabla 5). En reuniones se evalúa la manera como la organización administra su información de valor entre sus propios miembros, cómo se distribuye esta información, el acceso a la misma y la capacidad de transmisión de datos significativos (G3Pymes, 2009).

Tabla 5. Resumen de variables del componente comunicación e información v9D

\begin{tabular}{|l|c|}
\hline Reuniones & 59,44 \\
\hline Información, datos y conocimiento & 44,74 \\
\hline Indicadores pragmáticos TIC & 82,14 \\
\hline
\end{tabular}

Fuente: elaboración propia.

Aunque la cantidad de variables que contempla este elemento en el MMGO v10 es menor, hay que resaltar que estas perciben con más eficiencia la información que su contraparte en la versión 9D. El nuevo componente sintetiza de forma apropiada variables que en la versión anterior estaban definidas de modo demasiado específico y que, al final del ejercicio, afectaban el resultado estadístico. La implementación de la v9D mostró este componente como una fortaleza de la organización, pues obtuvo alta puntuación en aspectos demasiado específicos y dar 
al mismo una ubicación preferencial como el principal de ellos dentro de la organización. En la aplicación del v10, se orientaron con más precisión los resultados del factor comunicación e información, se llegó a un concepto efectivo dentro de la realidad actual de la empresa y se excluyó de la lista de las principales fortalezas. Estas razones sirven de base para validar este componente actualizado en la versión 10 del MMGO.

\subsubsection{Componente de responsabilidad social}

Con este componente, la v10 del MMGO evalúa la existencia de una estrategia de importaciones definida y explícita, los insumos de información sobre los proveedores externos, la existencia de insumos importables y cómo la empresa mide y recibe realimentación de esos proveedores para la toma de decisiones interna (tabla 6) (G3Pymes, 2013).

Tabla 6. Resumen de variables del componente responsabilidad social $\mathrm{v} 10$

\begin{tabular}{|l|c|}
\hline Enfoque estratégico de la RSE & 37,50 \\
\hline Gestión de la RSE & 41,67 \\
\hline Impacto de la RSE & 8,33 \\
\hline
\end{tabular}

Fuente: elaboración propia.

En la v9, este elemento examina las políticas relacionadas con la inversión social, antisoborno y anticorrup- ción y protección de la propiedad intelectual (tabla 7). Asimismo, se considera lo relacionado con los registros y documentos que sirven de soporte al sistema para evaluar su implementación (G3Pymes, 2009).

Tabla 7. Resumen de variables del componente responsabilidad social v9D

\begin{tabular}{|l|c|}
\hline Protección de la propiedad intelectual & 13,89 \\
\hline Políticas de inversión social & 16,67 \\
\hline Políticas antisoborno y anticorrupción & 8,33 \\
\hline Registros y documentos & 8,33 \\
\hline
\end{tabular}

Fuente: elaboración propia.

En la versión anterior del MMGO, las variables de este componente estaban enfocadas a las políticas y la documentación de la responsabilidad social. Esta es una apreciación algo limitada y, al poseer solo cuatro variables, puede arrojar resultados estadísticos significativos en el análisis final de los resultados, como sucedió en la aplicación de estos modelos en Compusistems, en donde, según el MMGO v9D, es un componente crítico y la principal debilidad de la empresa, al considerar variables de políticas y registros con puntajes muy bajos. Por su parte, la versión 10 contempla aspectos de la responsabilidad social de forma sistémica, vista desde la estrategia organizacional, su gestión e impacto. Esto redunda en resultados relevantes en el ejercicio de la consultoría 
y motiva la validación del componente de la versión más reciente del modelo.

\section{CONCLUSIONES}

La aplicación del modelo MMGO en sus versiones 10 (nueva) y 9D (anterior) se realizó en la empresa Compusistems, la cual fue un terreno de investigación propicio para esta labor. El gerente general participó activamente y facilitó la información y las instalaciones de la organización. Lo único que solicitó a cambio fue mantener el anonimato y el carácter de confidencialidad en cuanto a su nombre y al de la pyme y a la información provista por la compañía. Esto se ha cumplido mediante el uso de un pseudónimo en la presentación de los resultados del estudio.

Luego de realizar las comparaciones y los análisis en cada uno de los tres componentes actualizados del MMGO v10 frente a la versión anterior, junto con el análisis comparativo de los informes integrales, fue posible validar la nueva versión del modelo, en particular, de los componentes actualizados: análisis del entorno, comunicación e información y responsabilidad social empresarial.

Se concluye que el componente de análisis del entorno, propuesto en la nueva versión 10 del MMGO, no es viable, porque no sopesa aspectos del entorno que la organización suministra durante el proceso de recolección de información y que son relevantes para el ulterior proceso de diagnóstico. Además, en términos de practicidad para el investigador, no es conveniente, pues no es muy amigable hacer el análisis de la información recabada, algo que sí sucedía en la versión 9D.

Las actualizaciones de los componentes comunicación e información y responsabilidad social empresarial son apropiadas y acertadas en la versión 10 del MMGO, porque sintetizan con eficiencia las variables que tenía la versión anterior y que alteraban los resultados evidenciados en los informes integrales.

Así, como corolario de este estudio de validación del MMGO versión 10 en una pyme en la ciudad de Bogotá, se sostiene que el componente de análisis de entorno del MMGO v9D es apropiado para ser incluido en la versión 10. Los componentes actualizados de comunicación e información y responsabilidad social empresarial propuestos en la v10 deben conservarse, por los adelantos que traen consigo y las ventajas que su uso implica.

\section{REFERENCIAS}

Dinero. (2014). Oportunidades y retos del 4G. Dinero.com. Re- 
cuperado de http://www.dinero. com/empresas/articulo/el-retoinstalar-4g/180872E

Escuela de Administración de Negocios [EAN]. (2014). Investigar con la Universidad EAN. Recuperado de http://investigacion. ean.edu.co/seccion/inicio.html G3Pymes. (2009). Modelo de modernización de la gestión para organizaciones. Bogotá: EAN.

G3Pymes (2013). Modelo de modernización de la gestión para organizaciones $v$ 10. Bogotá: EAN. Hernández, M. (2014). Industria TIC colombiana necesita muchos más ingenieros. Eltiempo.com. Recuperado de http://www.eltiempo.com/archivo/documento/ CMS- 13480380

Martín, J. (2013). Colombia necesitará más de 12 mil profesionales TIC en 2015. Recuperado de http://pulsosocial.com/2013/04/ 18/colombia-necesitara-mas-de12-mil-profesionales-tic-en-2015/ Ministerio de Tecnologías de la Información y las Comunicaciones [MinTIC] y Federación Nacional de Software y Tecnologías de la Información [Fedesoft]. (2012). Estudio de salarios y profesionales del sector de software y TI de Colombia. Bogotá: MinTIC y Fedesoft. Recuperado de http:// www.fiti.gov.co/Images/Recursos/estudio-de-salarios-y-profesionales-del-sector-de-softwarey-ti-de-colombia-2012.pdf
Ministerio de Tecnologías de la Información y las Comunicaciones [MinTIC]. (2013a). Fortalecimiento de la industria TI. Recuperado de http://www.fiti. gov.co/?aspxerrorpath=/Estrategia_de_Fortalecimiento.htm

Ministerio de Tecnologías de la Información y las Comunicaciones [MinTIC]. (2013b). Agencia Nacional del Espectro (ANE). Recuperado de http://www.mintic. gov.co/portal/604/w3-propertyvalue-6179.html

Pérez, R., Garzón, M. \& Nieto, M. (2009). Análisis empírico de la aplicación del modelo de modernización de la gestión para organizaciones en pymes colombianas. Revista EAN (65), 77-106.

Pérez Uribe, R. I., Nieto P., M., Velázquez Contreras, A., Castellanos, G., Garzón Gaitán, M., Vargas, H. A. et al. (2009). $M M G O$ : Modelo de modernización de la gestión para organizaciones. Bogotá: Universidad EAN.

Portafolio. (2013). Las TIC ya representan el $6 \%$ del PIB del pais. Recuperado de http://www. portafolio.co/economia/tic-representan-el-6-del-pib-del-pais

Unión Internacional de Telecomunicaciones [UIT]. (2011). Tendencias de las telecomunicaciones. Visión general del mercado de las TIC: el sector móvil continúa en cabeza. Recuperado de http://www.itu.int/net/itunews/ issues/2011/03/04-es.aspx 